Meta

Journal des traducteurs

Translators' Journal

\title{
Apports du cognitivisme à l'enseignement de la créativité en traduction
}

\section{Ioana Balacescu et Bernd Stefanink}

Volume 50, numéro 4, décembre 2005

Pour une traductologie proactive - Actes

For a Proactive Translatology - Proceedings

Por una traductología proactiva - Actas

URI : https://id.erudit.org/iderudit/019828ar

DOI : https://doi.org/10.7202/019828ar

Aller au sommaire du numéro

Éditeur(s)

Les Presses de l'Université de Montréal

ISSN

0026-0452 (imprimé)

1492-1421 (numérique)

Découvrir la revue

Citer cet article

Balacescu, I. \& Stefanink, B. (2005). Apports du cognitivisme à l'enseignement de la créativité en traduction. Meta, 50(4). https://doi.org/10.7202/019828ar
Résumé de l'article

Considérée comme une "problem solving activity " (Guilford 1975), la créativité, démystifiée, fait partie du quotidien du traducteur. Victimes d'idées préconçues et erronées sur la notion de "fidélité ", beaucoup de traducteurs sont insécurisés face à leur créativité. Ils peuvent alors, comme en témoigne un de nos exemples, manquer de courage et jouer la carte de la stratégie du "playing itsafe ", ou bien, lorsque, comme dans un autre cas, leur statut social et professionnel leur donne une certaine assurance, garder leurs solutions créatives et revendiquer leur " trahison », toutefois sans pour autant essayer de trouver des légitimations à leurs solutions. Légitimations qui restent la plupart du temps au stade de « mécanismes de justification " ponctuels. Une analyse des besoins nous permet de montrer comment ces justifications hétéroclites et éparses peuvent venir s'intégrer dans un édifice théorique cohérent, s'appuyant notamment sur des fondements cognitivistes, susceptible de donner au traducteur le courage de sa créativité.
Ce document est protégé par la loi sur le droit d'auteur. L'utilisation des services d’Érudit (y compris la reproduction) est assujettie à sa politique d'utilisation que vous pouvez consulter en ligne.

https://apropos.erudit.org/fr/usagers/politique-dutilisation/ 


\title{
Apports du cognitivisme à l'enseignement de la créativité en traduction
}

\author{
IOANA BALACESCU \\ Université de Craiova, Craiova, Roumanie \\ Ioanadi@hotmail.com \\ BERND STEFANINK \\ Université de Bielefeld, Bielefeld, Allemagne \\ bstefanink@hotmail.com
}

\begin{abstract}
RÉSUMÉ
Considérée comme une «problem solving activity ( Guilford 1975), la créativité, démystifiée, fait partie du quotidien du traducteur. Victimes d'idées préconçues et erronées sur la notion de « fidélité », beaucoup de traducteurs sont insécurisés face à leur créativité. Ils peuvent alors, comme en témoigne un de nos exemples, manquer de courage et jouer la carte de la stratégie du «playing it safe », ou bien, lorsque, comme dans un autre cas, leur statut social et professionnel leur donne une certaine assurance, garder leurs solutions créatives et revendiquer leur « trahison », toutefois sans pour autant essayer de trouver des légitimations à leurs solutions. Légitimations qui restent la plupart du temps au stade de «mécanismes de justification » ponctuels. Une analyse des besoins nous permet de montrer comment ces justifications hétéroclites et éparses peuvent venir s'intégrer dans un édifice théorique cohérent, s'appuyant notamment sur des fondements cognitivistes, susceptible de donner au traducteur le courage de sa créativité.
\end{abstract}

\begin{abstract}
Considered as a "problem solving activity" (Guilford 1950), creativity is part of the translator's everyday life. Many translators, however, are insecure about their own creativity. They are victims of their erroneous, preconceived ideas about the concept of "fidelity" in translation. Our experimental groups show two kinds of reactions to that insecurity. The "semi-professional" learners tend to go back to a "playing it safe" strategy at the first sign of criticism. Translators with a certain position in society and with a certain "reputation" as authors have the necessary courage to stick to their creative problem solving, but with the awkward feeling of a betrayal which they heroically feel obliged to lay claim to, though they try with numerous explanations to show that it is not a betrayal. Their "mechanisms of justification" are partly false and can partly be integrated into a theory of translational activity which is mainly based on the results of cognitive research and which is liable to give the translator more security and courage with regard to his creativity.
\end{abstract}

MOTS-CLES/KEYWORDS

créativité, didactique de la traduction, cognitivisme, analyse conversationnelle, théorie de la traduction

\section{Une analyse des besoins du traducteur}

Pour pouvoir déterminer l'utilité d'un quelconque apport théorique à la pratique du traducteur, il faut commencer par examiner s'il existe un besoin en la matière et quelle en est la nature. Nous le ferons à l'aide de deux corpus qui se complètent. Le premier est la transcription du débat mené par un groupe de quatre « semi-professionnels » de l'Institut de traducteurs et interprètes de l'université de Bucarest, qui avaient pour tâche de négocier la traduction d'un texte anglais vers le roumain, avec pour finalité d'aboutir à une version commune. En complément de ce débat pris sur le vif et soumis aux principes de l'analyse ethnométholodologique conversationnelle, nous 
présenterons l'auto-témoignage d'un traducteur professionnel qui réfléchit spontanément et sans y avoir été invité sur son activité traduisante. Le premier cas illustre l'échec d'une tentative de solution créative face à un problème de traduction. Le second montre comment un traducteur, qui se croit infidèle, se sent obligé de revendiquer sa trahison, tout en cherchant malgré tout à la justifier par mille explications. Dans les deux cas les besoins qui en résultent sont ceux d'une autorité légitimatrice de la créativité en traduction. Cette autorité nous la trouverons dans une réflexion théorique cohérente qui s'appuiera sur les résultats de recherches cognitivistes.

\section{Corpus 1 ( «ca scoase din cutie ») : l'angoisse du traducteur créatif devant sa créativité et le jet de l'éponge par manque d'assurance et de courage}

Nos informateurs roumains doivent traduire un texte qui décrit l'émerveillement des Allemands de l'Est devant les richesses de l'Allemagne de l'Ouest qui s'offrent à eux après la chute du mur de Berlin.

\footnotetext{
Sleek new cars speed along straightened and repaved autobahns. Shiny service stations come equipped with well-stocked convenience stores and gleaming self-service restaurants. Enormous supermarkets, furniture stores and shopping emporiums dot the east German landscape, and giant cranes stand tall against the sky. Every seat is filled at Dresden's magnificent neo-classical opera house: comfortable burghers sip French champagne during the intermissions. Even in grimy Bitterfeld, a mining and chemicals centre notorious for its pollution, well-dressed women from a nearby retirement home gather for creamy coffee and gigantic pastries at a Swiss-owned coffee shop. (Newsweek, February 28, $1994: 14$ )
}

Le mot «sleek», dans le contexte «sleek new cars » leur pose un problème de traduction, notamment dans la mesure où il s'inscrit dans une isotopie des richesses occidentales qui crée une atmosphère de brillance, de progrès, de confort, qui traverse tout le texte et que les traducteurs sentent très bien, si l'on en croit leurs réactions (l'inf. 4 parle d'une « atmosphère de luxe » (1. 6061).

Aussi, leur première proposition de solution sérieuse qu'est l'adjectif « elegant», ne leur convient pas, parce qu'ils trouvent que le mot sleek « contient bien plus que elegant» (inf. 3, 1.17). Par la suite, dans une sorte de « brainstorming », ils proposent un nombre impressionnant de qualificatifs (resplendissant, brillant, reluisant, moderne, confortable, cher, extravagant, poli, superlavé, superarrangé, etc.), que, dans une deuxième étape, ils associent à des superlatifs, comme « des bottes reluisantes » (inf. 1, 1.34), pour finalement aboutir - sans doute par association avec les bottes qui sont reluisantes, parce que toute neuves, sorties de la boîte dans laquelle elles viennent d'être achetées - à une solution métaphorique, qu'ils évaluent comme « rendant presque toutes les nuances qu'ils viennent d'énumérer » (inf. 1, 1.42): «ca scoase din cutie » (littéralement : « comme sorti d'une boîte », une locution qui, hors contexte, correspondrait par ex., au français « brillant comme un sou neuf »). Ceci après avoir évalué, au passage, encore une fois la proposition de « elegant» (inf. 3, 1.36) pour la juger comme « trop sèche » (inf. 1,1.3) - jugement de l'informateur 1 , repris par l'inf. 3, qui renchérit que « c'est trop peu face au mot anglais » (1.38-39) - et la rejeter une fois de plus.

Et pourtant, il suffit que l'informateur 4 dise que « elegant» lui parait « suffisant pour créer cette atmosphère de luxe » (1.60-61) pour que, sans le moindre commentaire, le reste des trois informateurs impliqués dans la négociation de cette traduction s'incline et laisse tomber la solution métaphorique qu'ils avaient approuvée avec enthousiasme un instant plus tôt. Mais l'informateur 3 qui, dès le départ, a évalué « sleek» comme étant « plus que elegant» (1.18), et qui revient à la charge en déclarant, juste avant d'arriver à sa trouvaille, que « elegant est trop peu face au mot anglais » (1.38-39), ne semble pas vraiment convaincu. Le fait qu'il exprime la volte-face qu'il opère à l'aide d'un phraséologisme, de surcroît en langue anglaise (dans une discussion qui a lieu en roumain) - «I can live with that » (1.62) - marque une certaine gêne ${ }^{1}$, comme s'il avait honte de s'être laissé emporter par son enthousiasme vers une solution aberrante, dont il veut se distancier, parce qu'il n'a aucun argument logique pour la défendre.

Ce qui est frappant, c'est que l'informateur 4, qui condamne la solution métaphorique, est le seul qui n'ait pas contribué le moindre mot à toute la négociation de cette traduction jusque là, ce 
qui laisse penser qu'il ne s'est pas impliqué au même degré que les autres dans l'opération traduisante. Son manque d'implication dans le débat a entraîné le manque d'empathie nécessaire pour adhérer à une solution métaphorique.

$\mathrm{Ne}$ retrouvons-nous là l'attitude du critique de traduction professionnel qu'est le lecteur dans une maison d'édition, qui n'a pas le temps de s'impliquer dans le texte autant que le traducteur et ne peut, de ce fait, développer le même degré d'empathie ? Nous nous référons là au témoignage de la traductrice d'Astérix, Gudrun Penndorf, qui, dans une interview, a reproché au lecteur de la maison d'édition allemande d'avoir changé certaines de ses propositions de traduction, propositions dont nous lui avions reproché le manque de cohérence textuelle, avant d'apprendre qu'il s'agissait de corrections de la maison d'édition. Il s'agit donc d'un problème qui concerne autant les apprentis-traducteurs que les professionnels de la traduction, l'aspect didactique autant que l'aspect commercial.

\section{Corpus 2 : La « trahison assumée » et revendiquée sur fond de « fidélité » mal comprise}

Et, lorsque le professionnel n'est pas soumis au jugement de la maison d'édition devant laquelle il doit défendre ses propositions, il n'en reste pas moins avec la conscience de « trahir » le texte et souvent « désarmé » face à son auto-évaluation critique de sa traduction, si on ne lui fournit pas les « armes » pour la défendre, comme en témoigne l'auto-témoignage suivant, dû à Ghjacumu Thiers, professeur d'université et linguiste, qui, malgré son aveu d'une trahison - qu'il revendique fièrement, parce qu'il se sent « désarmé », pour la justifier - fournit néanmoins, dans la suite de son article, toute une série de justifications pour légitimer sa créativité traduisante et se déculpabiliser. Lisons son auto-accusation!

Le charme particulier de la traduction vient toujours de ce qui m'apparaît comme la difficulté en même temps que l'enjeu d'une pratique pour moi exclusivement empirique. Celui-ci se manifeste lorsque je relis la version corse que je viens d'achever d'un texte poétique. Cette impression provient souvent du constat d'un écart irréductible entre l'expression dans le texte-source et celle qui intuitivement s'est imposée à moi, dans ma langue. C'est précisément la conscience de cet écart qui me satisfait. Une trahison involontaire d'abord, mais assumée par la suite dans l'absence de tout repentir, voire avec le sentiment d'une illumination. Ou d'une trouvaille, si l'on veut faire moins exalté. La relecture recommencée me conforte dans cette délicieuse erreur. Quelque chose comme la conscience d'un forfait réussi. Une illusion sans doute, mais comme elle donne envie de récidiver! Au prix de ce plaisir-là, la fidélité serait bien ennuyeuse. C'est pourquoi je me sens à la fois complètement désarmé et irrésistiblement attiré par la pratique de traduction (c'est nous qui encadrons). (Thiers $2003: 362$ )

L'opération à laquelle nous venons de procéder fournit la base de ce qu'en didactique on appelle une analyse des besoins. Examinons la nature de ces besoins!

\section{Un besoin de légitimation par la théorie}

Malgré sa proclamation de l'inutilité d'une théorie pour le traducteur - proclamation qui est répétée tout au long du recueil de réflexions sur la traduction poétique dont fait partie l'article en question Thiers n'arrête pas de fournir des explications pour justifier ses «trouvailles » par des réflexions d'ordre théorique. Le défaut de ces réflexions, c'est que d'une part, elles ne font pas partie d'un système argumentatif cohérent et, d'autre part, qu'elles sont d'ordre strictement linguistique et destinées à prouver la justesse des « équivalences » qu'il pu établir entre des unités de traduction. Pour cela Thiers n'hésite pas à faire appel à l'évaluation des sonorités entre les mots de la langue source et ceux de la langue cible, comparant le mètre des vers français et celui de leurs correspondants corses, etc., jusqu'à se laisser aller à des considérations étymologiques. Bref, un ensemble de de justifications hétéroclites qui devraient être transcendées pour arriver à une explication d'ordre plus général, à un niveau supérieur. Le chemin qui mène à l' " illumination »est, en effet, pavé d'enchaînements associatifs, comme ils ont été étudiés de façon systématique par les 
chercheurs associationistes en cognitivisme, comme par exemple Mednick (1962) avec ses RATs (Remote associates Tests), Lakoff (1987) avec ses « chainings », le chercheur sur la mémoire Schank (1982) et ses MOPs and TOPs, ou encore, pour ce qui est des sonorités et du rythme, Aitchison (2003).

On le devine : les explications de Thiers sont en grande partie pertinentes, mais auraient besoin d'un support théorique cohérent qui lui ferait prendre conscience que les solutions créatives ne sont pas des trahisons, mais qu'il est passionnément fidèle au sens du texte source tel qu'il le perçoit, dans la bonne tradition herméneutique, où le traducteur doit être avant tout fidèle à lui-même et traduire ce qu'il comprend (Stolze 2003).

\section{Considérations épistémologiques : priorité de l'approche intuitive et associative}

Voyons dans quel cadre théorique viennent se situer les réflexions deThiers, naïves du point de vue traductologique. En ce qui concerne la saisie du sens, Thiers écrit : « je traduis donc toujours à partir d'une impression et d'une intuition» (Thiers $2003: 364)^{2}$. Même son de cloches dans le commentaire de la deuxième traduction faisant suite à « animal vulatil », quand la solution créative est trouvée par «association d'idées » (Thiers 2003 : 372), ce qui correspond aux théories associationnistes (Mednick 1962) de la saisie du sens, considérée par certains traductologues (dont nous partageons les convictions ) comme une stratégie traduisante légitime (Risku 1998 : 154). Ceci toutefois à condition qu'elle fasse l'objet d'un contrôle subséquent par l'analyse textuelle (ce que Hönig 1995 appelle le «monitoring »). Cette démarche intuitive constitue un parti pris théorique face aux approches traduisantes basées sur la «Textlinguistik», qui plaident vigoureusement en faveur d'une analyse du texte exhaustive « avant » (!) la moindre tentative de traduction, ce qui est contraire à toute pratique professionnelle ${ }^{3}$. Et quand Thiers poursuit ses explications en écrivant : « Je découvre la cause de cet écart singulier. La proximité phonique des mots [...]», il formule naïvement ce que la linguiste cognitiviste Aitchison a prouvé par ses recherches sur la façon dont les mots sont stockés et activés dans notre cerveau : l'importance de la structure phonique des mots. Sans doute la lecture de Aitchison (2003) aurait été d'un grand secours dans l'analyse rétrospective à laquelle procède Thiers, puisqu'elle lui aurait permis de préciser, par exemple, qu'un des facteurs les plus importants dans l'établissement de liens associatifs entre le mots est le « rhythmic pattern of the words » (Aitchison 2003 : 141) et que « The relationship of strength between the various syllables is more important than the exact amount of stress on each one. » (Aitchison 2003 : 141). De même ces recherches de Aitchison auraient permis à Coco $(2003)^{4}$, un autre traducteur de poésie, présent dans ce volume, de s'exprimer de façon constructive et circonstanciée, plutôt que de crier haro sur les incultes qui ignorent le décasyllabe : «E inammissibile tradurre un sonetto di Gongora, senza sapere cos'e un endecasillabo »(Coco 2003 : 134).

\section{La métaphore, source de compréhension et créatrice de sens}

Position théorique également lorsque Thiers exprime sa conception du traducteur comme créateur et non pas comme reproducteur, qui était le point de vue des approches structuralistes. Passons sur les remarques linguistiques de Thiers concernant le rôle de l'étymologie, des sonorités, etc. impliqués dans le déclenchement de la « trouvaille », qui confirment en partie les découvertes des cognitivistes, pour en venir à sa conception de la métaphore : «La métaphore de la langue conservée, mais modifiée, joue un rôle primordial dans cette manière, très concrète, de se représenter la vie qui ne peut s'accommoder d'un patrimoine linguistique fini » (notre mise en relief). Ne retrouvonsnous pas là les considérations de Lakoff/Johnson (1980: 117): « [...] metaphors allow us to understand one domain of experience in terms of another » et «Such metaphors are capable of giving us a new understanding of our experience. [...] highlighting some things and hiding others » (Lakoff/Johnson 1980 : 139) ? Le fait que ces métaphores « [...] are grounded in correlations within our experience » (Lakoff/Johnson 1980 : 154-155), et non pas isolées dans notre mental $^{5}$, explique les associations (la stratégie associationniste) dans $1^{6}$ approche du sens. Et, comme les structures conceptuelles varient d'une culture à $l^{\text {' }}$ autre, cela explique le fait qu'on traduise une métaphore par une autre ou qu'on élargisse ou «modifie » une métaphore, tel que 
l'entend Thiers, puisque, dans la traduction, elle viendra s 'intégrer dans un autre système conceptuel et prendra une autre «valeur» (au sens Saussurien du terme) : «But the human aspects of reality are most of what matters to us, and these vary from culture to culture, since different cultures have different conceptual systems. » (Lakoff/Johnson 1980 : 146).

\section{Bilan provisoire: il $\mathbf{n}^{6} \mathbf{y}$ a pas de tête traduisante vierge de théorie !}

Que nous apprend l'analyse de ces deux exemples?

Elle nous apprend qu'il n'y a pas de tête traduisante vierge de théorie. Les théories sont simplement sous-jacentes et déterminent les comportements traduisants de façon inconsciente. Il faut être très sûr de soi pour ne pas se laisser ébranler par les reproches de traîtrise, lancés par les

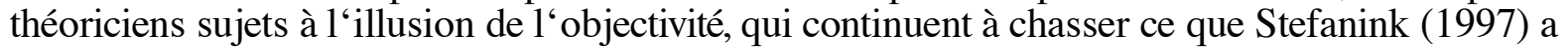
appelé « le fantôme de 1'objectivité ». Même des traducteurs chevronnés, praticiens excellents et trouveurs de «trouvailles » qui emportent le lecteur, ne sont pas à 1'abri des critiques d'un éditeur,

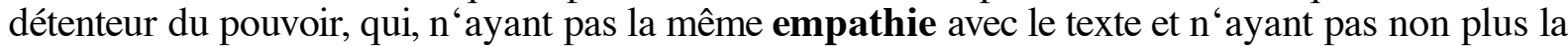
largesse d'esprit que lui assurerait une théorie de la traduction née de la pratique, se permettra de remplacer les trouvailles par des fabrications plus « conformes ». Si la traduction poétique fait peutêtre exception, parce qu'on n'osera pas s'attaquer au poète-créateur, sacré par ses réussites en langue maternelle, on s'acharnera d'autant plus sur des traducteurs d'œuvres moins sacrées, comme par exemple Astérix, qui sollicitent tout autant la créativité du traducteur et qui attireront

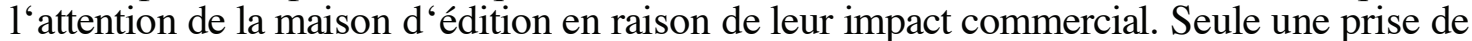
conscience des maximes de traduction inconscientes qui conditionnent sa pratique et une réflexion structurée sur les facteurs à prendre en considération lors de son activité traduisante peuvent donner au praticien l'assurance nécessaire pour se défendre face au reproche de traitrise lorsqu'il trouve une solution créative à un problème de traduction. Et c'est cette réflexion structurée qui constitue ce que nous appelons une approche théorique. Ce n'est donc pas un ensemble de règles, de type algorithmique, censées mener à la (!) traduction objective, à laquelle les « objectivistes » vont délivrer leur label de qualité, mais une prise de conscience des facteurs impliqués dans l'opération traduisante.

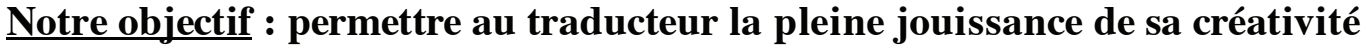

On entrevoit peut-être un peu notre objectif : c'est de donner au traducteur, condamné à la créativité du fait des barrières culturelles qui se manifestent dans la langue, le courage et la pleine jouissance de sa créativité, surtout s'il en est encore à ses débuts. Car les explications données à la suite des solutions créatives trouvées par les traducteurs-poètes, que nous venons de commenter, trahissent bien un certain besoin de légitimation. Et cette légitimation, c'est une réflexion théorique cohérente qui la lui fournira. Cette réflexion devra dépasser le stade des microstructures qui, comme le montrent nos traducteurs-poètes, laissent un sentiment d'insatisfaction car, comme le souligne Risku (1998 : 220), il s'agit plutôt de «mécanismes justificatifs » ( «echtfertigungsmechanismen ») que de «stratégies solutionneuses de problèmes » $(« \text { Problemlösungsstrategien } »)^{6}$.

\section{Le courage de la créativité grâce à la connaissance du processus cognititif}

En revanche, un deuxième exemple montre comment un groupe d'étudiants, qui a été initié aux résultats des recherches cognitivistes ainsi qu'à leur apport à la traductologie, a trouvé des solutions créatives à des problèmes impossibles à résoudre par la pensée «convergente », d'ordre analytique.

\section{Le corpus « Marianne »}

Si la France n'était pas Femme, comment pourrait-on officiellement lui annoncer qu'elle est devenue Veuve, comme l'a fait un matin le nouveau Président de la République ?

Imaginons un instant les possibilités de veuvage pour les autres nations. 
Sont à éliminer, tout d'abord, tous les pays qui, du fait même de leur sexe, ne sauraient être veuves. Non seulement veuf ne fait pas le poids de crêpe, mais il n'engendre pas le même désespoir. Imagine-t-on les EtatsUnis, le Royaume-Uni, le Canada, Le Mexique s'entendre dire qu'ils sont veufs ? Voit-on le Pérou veuf ? Rubbish!, on prend les nations féminines, l'image ne <colle> pas davantage : en annonçant « L'Allemagne est veuve » on risque de n'attendrir personne. « L'U.R.S.S. est veuve » ... ça paraît énorme. La Chine... on n'ose pas y penser. Quant au Royaume-Uni, même en changeant de sexe - je me sens gêné d'émettre cette hypothèse - je ne puis franchement supposer que quelqu'un, même haut placé, puisse annoncer à la GrandeBretagne qu'elle est Veuve. Miss Britannia est pour le monde entier une célibataire endurcie qui n'a jamais eu, à notre connaissance, d'autres liaisons que maritimes. Il y a au surplus dans le mot même de veuve une tendresse tout à fait étrangère au caractère inflexible et à la nature coriace de notre image de marque. Sans parler du volume de voiles qui s'accorderait mal avec la sobriété du deuil anglican. (Daninos : Les Nouveaux Carnets du Major Thompson, pp. 16-17)

La difficulté de ce texte, dont l'humour est basé sur les différences de genre grammatical des noms de pays, réside dans le fait que ces différences ne sont pas les mêmes dans d'autres langues et que le traducteur doit compenser ce manque de parallélisme.

\section{Les étapes de la créativité}

La façon dont nos informateurs du corpus Marianne abordent les problèmes posés par la traduction de ce texte montre qu'ils ont bien assimilé et mis en application les contenus de leur enseignement théorique.

Les traducteurs y arrivent en suivant un processus de concrétisation par antropomorphisation, qui est introduit par la remarque de l'informateur 2 «wir müssen irgendetwas, z.B. Herren, Damen reinschieben » (= il faut introduire quelque chose comme Messieurs, Dames). Ensuite on assiste à un processus de concrétisation, dû à une visualisation de différentes " scènes » possibles, associées avec ce mot. Cette visualisation s'appuie sur notre bagage cognitif, c'est-à-dire, dans ce cas, les clichés stéréotypés que nous véhiculons par rapport aux pays dont il s'agit. Ceci se passe chez nos informateurs avec une conscience aigüe du problème, qui est précisé à plusieurs reprises, en passant par des étapes, qui sont en fait celles décrites habituellement par les chercheurs en créativité.

\section{La sensibilité au problème}

Dans la conception de la créativité, comme «problem solving activity », proposé par Guilford, une des premières qualités de l'individu créatif est la sensibilité au problème à résoudre : « sensitivity to problems » (Guilford 1950). Dans la tradition herméneutique allemande celle-ci est intimement liée au terme de «Achtsamkeit », que Gadamer a emprunté à Heidegger et que les chercheurs en créativité comme Brodbeck (1999 : 58-67) ont placé au centre de leurs réflexions ${ }^{7}$. Les informateurs de notre Corpus Marianne font preuve de cette sensibilité au problème, dont ils prennent conscience dans les termes suivants ${ }^{8}$ :

Das ist ja die entscheidende Stelle: im Deutschen ist das mit dem Geschlecht nicht so ( c'est là le passage décisif : en allemand ce n'est pas la même chose avec le genre » (lignes 86-87).

\section{Un élément de la «Skopostheorie » : la prise en considération du récepteur du texte :}

Cette prise de conscience est suivie d'une première proposition de solution, introduire «messieurs » et «mesdames » : «il faut introduire quelque chose, Messieurs, Dames » (92), qui est suivie d'une précision plus détaillée du problème - «tu peux introduire Messieurs, Dames, mais il faut quelque chose qui établisse un rapport avec l'image des pays ») (93-94) - avec prise en considération du récepteur en langue cible : 
der deutsche Leser [...] muss das mit den Ländern verbinden können und nachvollziehen können, warum das eine Frau ist und das andere keine Frau ist (le lecteur allemand doit pouvoir établir un rapport avec les pays et comprendre pourquoi ça c'est une femme et ça ce n'en est pas une) (97-99).

Suit une longue discussion sur le statut du lecteur, que certains voient comme un «Durchschnittsleser» (lecteur moyen) (136), alors que d'autres, plus circonstanciés, se détachent du cliché du lecteur moyen et y voient «irgendein intellektueller Sack » (un quelconque intellectuel) (138). C'est cette hypothèse d'un lecteur d'un certain niveau intellectuel qui suggère d'introduire, en français dans le texte allemand, «La Grande Nation », qui est effectivement un cliché phraséologique avec lequel les Allemands d'un certain niveau culturel s'accordent pour être représentatif des associations avec la France (avec un soupçon d'ironie amusée dans la voix), véhiculées par les Allemands. Proposition écartée une fois de plus probablement par manque de cohérence avec l'isotopie de l'antropomorphisation qui traverse le texte.

\section{Un autre élément de la « Skopostheorie » : la prise en considération de la typologie des textes}

Lorsque l'inf.3 (les inf. 3 et 4 sont moins créatifs que 1 et 2 et sont loin d'avoir assimilé aussi bien que 1 et 2 le contenu de l'enseignement théorique) propose d'expliquer entre parenthèses qu'en français le mot « France », à la différence de l'allemand Frankreich a le genre féminin, l'inf. 1 lui répond :

« du kannst aber nicht in so einem humoristischen Text so was in Klammern schreiben, das wäre gar nicht lustig $[\ldots] »$ (mais tu ne peux pas, dans un texte humoristique, mettre quelque chose de ce genre entre parenthèses, ce serait pas drôle du tout [...]) (106-108)

\section{Nihil ex nihilo!}

Éduquer à la créativité, c'est faire prendre conscience aux apprenants que la solution créative à laquelle ils sont parvenus n'est pas due à leur imagination vagabonde, qui les aurait amenés à « trahir » le texte, mais à une empathie intime avec le texte que l'herméneute Gadamer a appelée «Horizontverschmelzung » (la fusion des horizons), terme que les cognitivistes ont démystifié en nous faisant entrevoir à quel degré notre compréhension d'un texte est le résultat d'une interaction entre des processus top down et des processus bottom up, les premiers étant liés à notre vécu et à la structuration engrammatique de notre cerveau qui en résulte et qui conditionne notre compréhension, les seconds faisant partie de ce que Lederer (1994 :37) appelle « le contexte cognitif, constitué par les connaissances acquises à la lecture du texte, conservées en mémoire à court terme et servant à l'interprétation des segments de texte suivants ». Notre exemple nous montre d'ailleurs que, contrairement à ce que semble suggérer la définition de Lederer, le contexte cognitif ne sert pas seulement à l'interprétation des segments de texte suivants, ce qui suggère une démarche linéaire du traducteur, mais il sert aussi à des retours en arrière et à des corrections et ajustements d'une première compréhension tentative. Du point de vue du processus bottom up la compréhension du texte est en effet une démarche concentrique, fondée sur les structures isotopiques du texte. C'est lorsqu'il aura compris cette vérité fondamentale et la relation entre ces processus bottom up et top down que le traducteur ne se sentira plus comme un traitre et aura le courage de sa créativité.

Dans notre cas précis il faut faire comprendre à nos traducteurs que s'ils ont choisi Marianne et non pas « la République Française » ou «La grande Nation»-ce qui aurait également été une solution au problème du genre grammatical posé dans ce texte, solutions auxquelles ils ont pensé et qu'ils ont rejetées - c'est que le texte est traversé par une isotopie de l'antropomorphisation qui soumet le processus de compréhension à des contraintes sémantiques. Les supports de cette isotopie sont des mots comme « veuve », «attendrir », (on n'attendrit pas une république, qui est quelque chose d'abstrait, mais on attendrit Marianne), «tendresse », « Miss Britannia », etc. 
Lorsque, au cours de leurs débats ils utiliseront, un peu plus tard, le mot «stimmig » (cohérent) :

- aber erst mal müssen wir doch prinzipiell das Geschlechtsproblem lösen es muss alles stimmig werden (mais avant tout nous devons résoudre le problème fondamental du genre, il faut que tout soit cohérent) - (160-162),

ils montrent qu'ils sont bien conscients du problème et des critères textuels à observer pour le résoudre. Cette conscience vient de l'apport théorique dont ils ont bénéficié tout au long des séances de traduction qui ont précédé la traduction de ce texte; elle réduit à néant toutes les objections de ceux qui prétendent que la théorie ne sert à rien et qu'on ne peut enseigner la créativité, qu'ils considèrent comme quelque chose de mystérieux. Ceci ne veut pas dire qu'on ne peut arriver à cette solution spontanément, sans enseignement théorique, mais c'est ensuite que le problème se pose. Les chercheurs en créativité savent qu'après les phases de préparation, d'incubation et d'illumination vient la phase d'évaluation du produit créatif. Et c'est là que les informateurs de notre premier exemple ont flanché à la moindre esquisse de critique et ont repris leurs billes pour miser sur la sécurité. S'ils l'ont fait, c'est qu'on ne leur avait pas donné contrairement aux informateurs du groupe Marianne - les « armes » nécessaires pour légitimer leurs solutions créatives, autant face à eux-mêmes que face à autrui. Ils sont « désarmés » face à leur créativité, comme le dit explicitement Thiers (2003:362), dans l'exemple cité au début de cet article.

\section{La méthode : sur le mode du jeu}

La conscience de leurs démarches que nos informateurs ont acquise par leur apport théorique les amène à utiliser consciemment une stratégie du jeu :

Dann müssen wir es wie ein Spiel machen: hier Marianne und unten Uncle Sam oder so was (alors il faut faire comme si c'était un jeu : ici Marianne et plus bas Uncle Sam, ou quelque chose comme ça) (146-147)

et, plus bas :

Wir müssen das Spiel so weiterführen (il faut continuer le jeu comme ça) (171)

et encore :

Mit der Marianne, mit solchen Sachen, man spielt schon mit den Bildern, die man von diesen Ländern hat (avec Marianne et des choses comme ça, il est vrai qu'on joue avec les images qu'on a de ces pays) (225-226),

là encore une des stratégies qui ont fait l'objet de leur enseignement théorique, stratégie recommandée par des chercheurs en créativité, comme de Bono, dont le titre du livre Lateral Thinking a été traduit en allemand par Spielerisches Denken (= pensée ludique).

\section{Un critère fondamental : la « Stimmigkeit»}

Et c'est ainsi sur le mode du jeu - les nombreux rires qui viennent interrompre leur discussion en font foi - que nos informateurs trouveront Germania pour l'Allemagne après avoir, là encore, procédé à un choix très conscient par rapport à la solution également envisagée de « la République fédérale d'Allemagne » (179, solution qui serait également une solution au problème de la différence de genre), toujours dans l'optique du critère de la cohérence du texte (stimmig). Le monologue dans lequel se lance l'informateur 1, emporté par sa créativité, montre comment il est conscient de la cohérence du texte comme élément à prendre en considération, se référant, d'une part, à l'existence de Miss Britannia comme élément du processus bottom up dans le texte et, 
d'autre part, à l'élément Marianne, introduit par un processus top down, conditionné par la présence de Miss Britannia:

Es muss alles stimmig werden. Ja, deswegen wird es gehen mit der Germania, wenn wir hier die Marianne einführen; die ist ja das Gegenstück [...] Und im Prinzip ist das auch nicht schlecht, weil er führt die Miss Britannia an:Dieselbe Schiene. Super, das haben wir schon mal geklärt. (Tout doit être cohérent. Oui, c'est pourquoi cela va marcher avec la Germania, si on introduit ici Marianne; elle est en fait la contrepartie [...] Et en principe c'est pas mauvais parce qu'il mentionne Miss Britannia : le même principe. Super, voilà une chose de réglée) (161-168).

N.b. : il faut comprendre «stimmig » (cohérent) ici comme un terme introduit par Stolze (1992) dans l'approche herméneutique en traductologie en tant que critère principal à respecter lors d'une traduction. Les étudiants ont été familiarisés avec ce terme au cours de leur préparation ; c'est donc en connaissance de cause qu'ils l'utilisent ici comme critère primordial à respecter dans leur traduction

\section{Un des éléments du jeu : «fluency of thinking »}

Un des éléments de ce jeu qui mène à la solution créative est, selon les chercheurs en créativité, la fluidité de la pensée ( «fluency of thinking » Guilford 1950). Le fait que nos informateurs travaillent en groupe favorise évidemment cette fluidité et les amène à plusieurs séances de brainstorming spontanées au cours desquelles fusent les visualisations le plus saugrenues, mais aussi des solutions finalement adoptées ; ainsi ils aboutissent au « bûcheron canadien », en élargissant consciemment leur visualisation de la «scene » (sensu Fillmore) Canada qui leur avait d'abord suggéré l'image de la feuille d'érable.

\section{Un autre élément du jeu : la concrétisation des éléments de la « scene » visualisée}

L'objet de leur « pensée fluide » est très souvent une concrétisation de certains éléments de la «scene » visualisée, ceci dans le but de se rassurer sur la pertinence de la solution trouvée, en lui fournissant des assises dans la réalité en faisant appel à son world knowledge (le « bagage cognitif » de Lederer 1994). Ainsi l'informatrice 2 veut absolument donner un visage encore plus concret à la France, déjà concrétisée par Marianne, en évoquant les noms de Catherine Deneuve (139), Laetitia Casta (193). Notons que dans les débats on a également mentionné le nom de Jeanne d'Arc (193), qui a été écarté par manque de cohérence avec le reste.

\section{Un modèle de comportement créatif réutilisable}

La traduction allemande du texte à laquelle sont parvenus nos informateurs montre comment ils ont appliqué les principes de la concrétisation antropomorphisante aux autres noms de pays qui posaient des problèmes du même type, respectant ainsi d'une part, dans un processus bottom up, cet aspect qui est un des éléments constitutifs du tissu textuel et interpellant, d'autre part, leur vécu, dans un processus top down, tout en étant conscients du respect qu'ils devaient à la cohérence textuelle et à la fonction du texte dans la bonne tradition d'une skopostheorie, issue de la linguistique du texte :

Wenn Frankreich nicht Marianne wäre, wie könnte man ihr dann offiziell verkünden - so wie es eines

Morgens der neue Präsident der Republik getan hat - dass sie Witwe geworden sei?

Stellen wir uns einmal die Möglichkeiten der Witwenschaft für die anderen Nationen vor.

Zunächst gilt es, all die Länder auszuschließen, die schon allein auf Grund ihres Geschlechts, keine

Witwen sein könnten. Nicht nur dass die Witwerschaft nicht das gleiche Gewicht an Trauerflor auf die

Waage bringt, sie ruft auch nicht dieselbe Verzweiflung hervor. Stellen wir uns vor es würde dem amerikanischen Cowboy, dem britischen Gentleman, dem kanadischen Holzfäller oder dem mexikanischen Caballero plötzlich erklärt, er sei Witwer geworden? Oder sieht man etwa Peru als Witwer an? Unsinn! 
Nehmen wir die „weiblichen“ Nationen, so passt das Bild auch nicht besser: Verkündete ma,n die deutsche Germania sei Witwe geworden, so würde man wohl kaum jemanden zu Tränen rühren. „Die Sowjet Union ist Witwe", scheint ungeheuer. China...man wagt nicht einmal daran zu denken. Und was schließlich das Vereinigte Königreich angeht, selbst wenn man das Geschlecht veränderte - es ist mir bei dieser Hypothese etwas unbehaglich zumute - so kann ich mir beim besten Willen niemanden vorstellen, nicht einmal aus den oberen Etagen, der Großbritannien verkündete, dass es Witwe geworden sei. Miss Britannia ist für die ganze Welt eine eingefleischte Junggesellin, welche, unsers Wissens nach, niemals andere Beziehungen gehabt hat, als jene maritimer Natur. Darüber hinaus gibt es im Wort „Witwe“eine gewisse Zärtlichkeit, die dem unbeugsamen Charakter und der hartnäckigen Natur unseres Image gänzlich fremd ist. Ganz zu schweigen von der Masse an Trauerflor, welche sich schlecht mit der Nüchternheit der anglikanischen Trauer vertrüge.

(Il s'agit de la version à laquelle est parvenue le groupe ; nous avons laissé les imperfections telles quelles)

\section{Le texte « palais »}

Dire que la créativité s'apprend veut dire qu'on peut trouver des modèles de comportement réutilisables dans d'autres contextes. C'est ce qui est arrivé, par exemple, lorsque quelques semaines plus tard, nos informateurs ont eu à traduire le texte suivant :

L'avouerai-je? (hypocrite interrogation qui retarde un aveu tout prêt) : lorsque, après avoir passé la journée à traquer le tigre royal en Assam, je me retire sous la tente face à mon plat de riz et à ma feuille de bétel, il m'arrive dans mon sommeil de faire des rêves de palais. Non pas du Taj Mahal ou - que ma Souveraine me pardonne - de Buckingham Palace, mais de rognons de veau aux morilles à la crème, tel que me les prépare la Mère Darsonval dans la vallée du Cousin.

Honni soit qui rognon pense !... Mes papilles soudain s'émeuvent et je suis capable, le lendemain, d'abandonner mon tigre pour rejoindre un petit coq au vin du pays de la grandeur. Le seul pays de l'univers où l'on dise d'un fromage qu'il est sincère, d'un vin qu'il a de la jambe, et qui trouve tout à fait normal de décerner les quatre étoiles d'un général de corps d'armée à un chef cuisinier.

(Daninos : Les Nouveaux Carnets du Major Thompson, pp. 6-7)

Une des diffcultés du texte était la traduction du jeu de mots basé sur le double sens de « palais ». Après avoir pris conscience du problème, ce qui se manifeste dans des phrases comme « il faut traduire le double sens, en allemand il n'y a rien », « il y a deux mots l'un c'est 'Palast' (c'est-àdire palais au sens de château) et l'autre c'est 'Gaumen' (palais en tant que partie de la bouche) », ils arrivent à la phrase suivante: " il faut trouver quelque chose qui associe en même temps 'Paläste' (plur. de Palast) et 'königliches Essen'(repas royal)». C'est-à-dire que, de même que dans l'exemple précédent, ils arrivent par un enchaînement associatif (le « chaining » de Lakoff) à partir de Gaumen à un élargissement de la scene visualisée (Fillmore), puis procèdent à un changement de focalisation (selon le figure/ground alignment de Langacker), passant de la visualisation de l'organe à ce que l'on mange avec cet organe : königliches Essen (repas royal).

S'ils arrivent au qualificatif de royal, c'est évidemment, là encore sous l'influence du contexte isotopique de la splendeur, c'est-à-dire dans un processus bottom up. Une fois l'expansion adjectivale « royal » trouvée, nos informateurs se lancent à nouveau dans un brainstorming incontrôlé, produisant une série de syntagmes, dans lesquels ils laissent libre cours à leurs associations avec « royal » : « mit königlichem Leben » (vie royale), « königliches Essen » (repas royal), « königlicher Wohnort » (logement royal), « königliche Besitztümer » (possessions royales), etc., ce qui correspond, une fois de plus, à l'une des stratégies qui, selon les chercheurs en créativité, doit mener à une solution créative (fluency of thinking).

Ce qui est frappant et ce qui montre que les attitudes qui mènent à des solutions créatives peuvent faire l'objet d'un enseignement et d'un apprentissage, c'est le fait qu'à un moment ils font appel à une expérience didactique antérieure : 
War es das letzte Mal hier oder?[...] Das war nämlich auch wieder so was, was man nicht übersetzen konnte, das war irgenwie so [...] (est-ce que c'était ici, la dernière fois ? [...] parce que là aussi il y avait quelque chose qu'on ne pouvait pas traduire, c'était quelque chose comme [...])

Ce qui provoque un autre informateur à formuler la démarche stratégique décisive:

Ja wahrscheinlich müssen wir das Wort Palast schon irgendwie reinbringen, aber das [...] Wahrscheinlich in so nem [...] zusammengesetzten Wort dann mit irgendwas [...] gaumenfreudigen artigem [...] (oui probablement qu'il faudra bien qu'on introduise le mot palais d'une façon ou d'une autre, mais probablement dans un $[\ldots]$ mot composé, avec quelque chose qui fasse partie de la nature des choses qui ont bon goût $[\ldots])$.

En parlant de « mot composé » ils évoquent un exemple de solution créative mentionné dans le cadre de l'apport théorique que nous leur avons donnée dans ce cours. Il s'agit de la traduction des paroles du barde Assurancetourix qui, dans le volume d'Astérix intitulé La rose et le glaive, réagit furieusement à la nouvelle qu'on a engagé une barde pour faire la classe de musique aux enfants du village en criant qu' " une barde ça n'existe pas, sinon ce serait une tranche de lard ! », jouant sur la double sémantique du mot «barde ». Nous avons montré comment la traductrice allemande, expoitant à merveille les possibilités de formation de mots composés,caractéristiques du génie de la langue allemande, a trouvé une traduction créative avec «Bardinnen gibt es nicht, höchstens Bardamen! », jouant sur le statut linguistique de la première syllabe de Bardinnen, associé avec un élément du mot composé Bardamen.

On peut donc constater que les apprenants ont non seulement été capables de trouver une solution créative pour un élément problématique du premier texte et d'appliquer ensuite cette solution avec cohérence à l'ensemble du texte, mais qu'ils ont réemprunté les voies de disponibilité créative ainsi créées pour résoudre des problèmes dans le texte numéro 2.

Ajoutons que ces voies créatives on été frayées dans un esprit tout à fait conforme aux préceptes des herméneutes et de leur conception d'une «Horizontverschmelzung », où les éléments du texte (notre Miss Britannia, du texte 1) viennent se fondre dans le contexte associatif du récepteur du texte (Marianne pour la France, Germania pour l'Allemagne etc.), tout cela dans le respect mutuel d'un équilibre entre processus bottom up et processus top down.

Et c'est très consciemment que nous utilisons l'image d'une «voie frayée ». Les cognitivistes nous aprennent, en effet, que ce sont les expériences répétées qui finissent par frayer des chemins, c'est-à-dire par créer des voies neuronales qui forment la base des structures associatives qui nous permettent d'aboutir à des solutions créatives. Plus nous faisons des expériences du même ordre, plus ces voies déjà frayées - dans un processus qu'on peut rapprocher des catégorisations qui sont à la base de toute compréhension - attirent ces expériences qui viennent les renforcer jusqu'à créer des structures engrammatiques. La façon dont nos informateurs ont résolu le problème du texte numéro 2 montre qu'une voie commençait à être frayée.

\section{Conclusion}

Ces exemples nous ont montré à quel point un enseignement théorique cohérent, notamment de nature cognitiviste, fournit une légitimation à la créativité sptonanée du traducteur et favorise son éclosion de façon systématique.

Lorsque le traducteur est conscient du fait que sa créativité commence dès sa perception du texte, qui est fonction de son vécu, comme nous l'apprennent les cognitivistes, et que ce vécu, conditionné par sa culture, a structuré son mental en réseaux engrammatiques à travers lesquels il perçoit la réalité de façon forcément différente de l'individu qui a vécu dans une autre culture, il se détachera aisément des « maximes de traduction » qui risquent de conditionner ses choix traduisants (comme par ex. : « trouver un mot qui rende tout », dans notre exemple roumain).

S'il est familiarisé avec la sémantique des «scenes-and-frames », il se laissera aisément aller à ses visualisations. S'il sait que ces scènes qu'il visualise ont une structure de «figure/ground alignment » (Langacker 1987 :120ss.) et que les éléments scéniques qui font partie du « ground» 
Sont susceptibles de devenir «figure » (puisque Langacker nous dit que la relation entre figure et ground n'est pas établie une fois pour toutes), il se laissera aisément aller à une énumération rapide des éléments scéniques, dont on sait qu'elle constitue une base propice à la créativité (Guilford 1975 :40, parle de «fluency of thinking »). Et si, finalement, il sait que dans la culture de la langue cible, d'autres éléments scéniques du « ground » peuvent venir jouer rôle de «figure », il ne sera plus influencé dans ces choix traduisants par une «maxime de traduction » qui lui dit, à tort, qu'il faut trouver en langue cible « un mot qui rende le tout du mot en texte source », mais sera prêt, par exemple, à procéder à un changment de focalisation pour trouver und solution créative.

Les exemples que nous venons de voir montrent comment l'apport cognitiviste vient compléter de façon idéale les éléments plus traditionnels de la linguistique du texte (prise en considération de la fonction du texte mise en évidence par la «skoposthéorie », de la typologie des textes, de l'importance de la conscience isotopique, etc., ...) pour former un ensemble didactique cohérent. Ils illustrent de façon convaincante l'introduction d'un enseignement théorique dans la formation du traducteur.

Dans la pratique enseignante cet enseignement théorique se constituera sur la base d'une analyse des besoins, telle que nous l'avons présentée, analyse qui s'effectuera, ensemble avec les participants, sur le corpus de données conversationnelles qu'ils auront fourni. Ensuite, au long des différents textes en voie de traduction, l'enseignant fera réfléchir ses étudiants sur les problèmes à résoudre, à la lumière de ce fonds théorique.

\section{Une remarque pratique : intérêt du travail en sous-groupes et composition des groupes}

L'atmosphère de travail dans un groupe est décontractée et se passe sur le mode du jeu, comme en témoignent nos enregistrements, où les débats sont fréquemment entrecoupés de rires. Ce sont là des éléments constitutifs de toute activité créatrice, selon des chercheurs en créativité comme de Bono. Évidemment le groupe doit être préparé à ce travail. À l'enseignant de créer une atmosphère de confiance, nécessaire à cette atmosphère ludique, en montrant par exemple que toute association peut donner lieu à d'autres qui finissent par aboutir à une solution créative acceptable au cours d'enchaînements associatifs souvent inattendus (sensu Lakoff 1987, qui parle de « chainings »).

Ce que nous avons pu remarquer dans nos corpus conversationnels, c'est que le non respect du critère d'homogénéité du groupe n'a pas l'effet négatif qu'on veut bien lui prêter. Ainsi dans notre corpus «Marianne » l'informateur 4, qui a du mal à suivre le mouvement créatif des informateurs 1 et 2, pose des questions, qui obligent l'informateur 1 à prendre conscience de leurs démarches et à formuler les problèmes et les moyens de les résoudre, ce qui entraîne une prise de conscience générale de la macrostratégie à suivre, principe fondamental, selon Hönig, à la base de toute stratégie traduisante et indispensable au processus de modélisation qui doit présider à toute opération traduisante. Cette prise de conscience est bénéfique pour l'ensemble du groupe.

\section{NOTES}

1. On pense à la fonction de l'introduction d'un élément langagier étranger dans la littérature, par ex. lorsque dans $A$ Farewell to Arms de Hemingway, le prêtre demande à Henry: «Are you croyant ? », une façon de surmonter la gêne provoquée par le caractère trop intime de la question.

2. Stefanink (1997) n'a pas hésité à conférer le statut de «Sinnerfassungsinstrument» (= instrument de saisie du sens) à l'intuition, parlant d'une « epistemologische Wende » (un tournant épistémologique) par opposition aux théoriciens qui prescrivent une analyse exhaustive du texte, afin d'en découvrir le sens par l'analyse, avant de céder à la moindre velléité de traduction, ce qui semble contraire à toute pratique professionnelle.

3. Cf. à ce propos Stefanink 1997, où il est question d'un « bouleversement épistémologique », et son plaidoyer pour une approche intuitive, l'analyse à posteriori ( !) des solutions trouvées servant uniquement la plausibilité interindividuelle.

4. Coco, Emilio (2003) : «Alcune precisazioni e riflessioni », dans Thiers (éd.), p. 130-153. 
5. «This suggests that understanding takes place in terms of entire domains of experience and not in terms of isolated concepts » (Lakoff/Johnson $1980: 117)$

6. « Ce qui est typique pour les méthodes des non-professionnels, c'est leur manque d'intégration dans l'ensemble du processus de traduction. Leur fonction tient plus de mécanismes justificatifs que de stratégies servant à résoudre des problèmes. C'est pourquoi elles peuvent facilement devenir des obstacles à la créativité ( Kreativitätshemmer») : le traducteur n'accepte pas ses propres propositions, même dans les cas où la communication en langue cible exigerait justement des solutions innovatrices et où la création personnelle conviendrait parfaitement à la situation de communication en langue cible» (Risku $1998: 220$ ) (notre traduction).

7. „Achtsamkeit“ est traduit par « attention» dans les dictionnaires. Mais à la différence de « Aufmerksamkeit» que les dictionnaires traduisent également par «attention », Achtsamkeit implique la participation de l'être entier. Stolze 2003 parle de la Leibhaftigkeit ('corporalité' rend mal le côté physique palpable auquel fait penser le mot allemand, c'est-à-dire la participation du traducteur avec tout son être), Aufmerksamkeit étant réservé pour une attitude liée à l'intellect.

8. Je traduis les données conversationnelles, la discussion a été menée en allemand. Les chiffres qui suivent les citations se réfèrent aux lignes du corpus

\section{RÉFÉRENCES}

Aitchison, J. (2003): Words in the Mind. An Introduction to the Mental Lexicon, Oxford, Blackwell. BALACESCU, I. (2004): « Le courage de la créativité », Le français dans le monde 334, p. 30-34.

BALACESCU, I. et B. STEFANINK (2001): « Une traductologie au service de la didactique : l'école allemande au sein de la famille traductologique, $1^{\text {ère }}$ Partie », Le langage et l'homme. Traductologie - Textologie XXXVI-1, p. 89-104.

BALACESCU, I. et B. STEFANINK (2002a): « Une traductologie au service de la didactique : l'école allemande au sein de la famille traductologique, $2^{\text {ème }}$ partie », Le langage et l'homme. Traductologie - Textologie XXXVII-1, p. 155 176.

BALACESCU, I. et B. STEFAnINK (2002b): „Die ,Wende’ in der Übersetzungswissenschaft und die sich daraus ergebenden Forschungsdesiderate“, in STEFAninK, B. and T. Fessenko (eds.): Reality, Language and Mind. An International Book of Researcher Reports, Tambov, Tambov University Press, p. 33-46.

BALACESCU, I. et B. STEFANINK (2003a): « Du structuralisme au cognitivisme: la créativité au fil des théories de la traduction », Le langage et l'homme. Traductologie - Textologie, Sciences du langage XXXVIII-1, p. 125-145.

BALACESCU, I. et B. STEFANINK (2003b): « Traduction et différences culturelles », Le français dans le monde 326, p. 21-35.

BALACESCU, I. et B. STEFANinK (2005a): « La didactique de la traduction à l'heure allemande », Meta 50-1, p. 277294.

BALACESCU, I. et B. STEFANINK (2005b): « Défense et illustration de l'approche herméneutique en traduction », Meta 50-2, p. 634-643.

BRODBECK, K.-H. (1999) : Entscheidung zur Kreativität, Darmstadt, Primis.

COCO, E. (2003): « Alcune precisazioni e riflessioni », in THIERS, G. (ed.): Baratti. Commentaires et réflexions sur la traduction de la poésie, coll. "Isule Literarie. Des îles littéraires», Albiana - Bu - Ccu - Iitm, p. 130-153.

GuILford, J. P. (1975): “Creativity: A Quarter Century of Progress", in TAYLOR, I. A. and J. W. GeTZEls (eds.): Perspectives in Creativity, Chicago, Aldine, p. 37-59.

HöNIG, H. (1995) : Konstruktives Übersetzen, Tübingen, Stauffenburg.

KußMaul, P. (2000): Kreatives Übersetzen, Tübingen, Stauffenburg.

LAKOFF, G. (1987): Women, Fire and Dangerous Things. What Categories Reveal about the Mind, Chicago,

University of Chicago Press.

LAKOFF, G. and M. Johnson (1980): Metaphors we live by, Chicago, University of Chicago Press.

LANGACKER, R. W. (1987): Foundations of Cognitive Grammar, Stanford, Stanford University Press.

LEDERER, M. (1994): La traduction aujourd'hui, Paris, Hachette.

Mednick, S. A. (1962): “The Associative Basis of the Creative Process", Psychological Review 69, p. 220-232.

RISKU, H. (1998): Translatorische Kompetenz: kognitive Grundlagen des Übersetzens als Expertentätigkeit, Tübingen, Stauffenburg.

SChank, R. C. (1982): Dynamic Memory. A Theory of Reminding and Learning in Computers and People, London/New York, Cambridge University Press. 
StefaninK, B. (1997): „'Esprit de finesse' - 'Esprit de géométrie': Das Verhältnis von 'Intuition’ und 'übersetzerrelevanter Textanalyse' beim Übersetzen”, in KELLER, R. (ed.): Linguistik und Literaturübersetzen. Tübingen, Narr, p. 161-184.

STEFAninK, B. (2000): « Analyse conversationelle et didactique de la traduction », Studia Romanica Posnaniensa 25/26, conférence tenue au colloque: Analyse des discours: méthodologies et implications didactiques et traductologiques. Poznan 7 - 10 juin 1998, Poznan, Adam Mickewicz University Press, p. 283-299.

STOLZE, R. (1992) : Hermeneutisches Übersetzen, Tübingen, Narr.

STOLZE, R. (2003) : Hermeneutik und Translation, Tübingen, Narr.

THIERs, G. (2003) : «L'écart parfait », in THIERS, G. (éd.) : Baratti. Commentaires et réflexions sur la traduction de la poésie, coll. «Isule Literarie. Des îles littéraires », Albiana - Bu - Ccu - Iitm, p. 363-372.

THIERS, G. (ed.) (2003): Baratti. Commentaires et réflexions sur la traduction de la poésie, coll. « Isule Literarie. Des îles littéraires », Albiana - Bu - Ccu - Iitm. 